The Astrophysical Journal, 689: L53-L56, 2008 December 10

(C) 2008. The American Astronomical Society. All rights reserved. Printed in U.S.A.

\title{
2MASS J09393548-2448279: THE COLDEST AND LEAST LUMINOUS BROWN DWARF BINARY KNOWN?
}

\author{
Adam J. Burgasser, ${ }^{1}$ C. G. Tinney, ${ }^{2}$ Michael C. Cushing, ${ }^{3}$ Didier Saumon, ${ }^{4}$ Mark S. Marley, \\ Clara S. Bennett, ${ }^{1}$ and J. Davy Kirkpatrick ${ }^{6}$ \\ Received 2008 August 25; accepted 2008 October 17; published 2008 November 20
}

\begin{abstract}
Determinations of the luminosity and atmospheric properties of the T8 brown dwarf 2MASS J09393548-2448279 are presented, based on Spitzer IRAC and IRS observations and ground-based astrometry. We find $\log _{10}\left(L_{\text {bol }} / L_{\odot}\right)=-5.69 \pm 0.03$ for this source, comparable to the current low-luminosity record holder 2MASS J04151954-0935066. However, modeling of near- and mid-infrared spectral data indicates an effective temperature of $600 \pm 35 \mathrm{~K}$, roughly $100 \mathrm{~K}$ cooler than $2 \mathrm{M} 0415$. These parameters require a highly inflated radius for $2 \mathrm{M} 0939\left(R \approx 0.13 R_{\odot}\right)$ which cannot be reconciled with brown dwarf structure models. However, if this source is an unresolved, equal-mass binary, then the reduced luminosity of each component $\left(L_{\text {bol }} \approx 10^{-6} L_{\odot}\right)$ can be brought into agreement with the inferred atmospheric parameters for an age of $0.4-12 \mathrm{Gyr}$ and component masses of 0.01-0.05 $M_{\odot}$. This hypothesis can be tested through future high-resolution imaging and/or spectroscopic observations.
\end{abstract}

Subject headings: binaries: close — stars: fundamental parameters stars: individual (2MASS J04151954-0935066, 2MASS J09393548-2448279) — stars: low-mass, brown dwarfs

Online material: color figures

\section{INTRODUCTION}

Wide-field, near-infrared (NIR) sky surveys have uncovered very low luminosity brown dwarfs whose low-temperature atmospheres host abundant gas and condensate molecular species. These include the $\mathrm{T}$ dwarfs, the latest known spectral class of brown dwarfs characterized by strong $\mathrm{H}_{2} \mathrm{O}, \mathrm{CH}_{4}$, and $\mathrm{NH}_{3}$ absorption in their infrared spectra (e.g., Burgasser et al. 2006b; Cushing et al. 2006). The latest type $\mathrm{T}$ dwarf with a reported parallax measurement is the T8 2MASS J04151954-0935066 (hereafter 2M0415; Burgasser et al. 2002; Vrba et al. 2004), for which Saumon et al. (2007) determine $T_{\text {eff }}=750 \pm 25 \mathrm{~K}$ and $\log _{10}\left(L_{\text {bol }} / L_{\odot}\right)=-5.67 \pm 0.02$ based on broadband spectral and photometric measurements and spectral model fits. A handful of even colder $\mathrm{T}$ dwarf candidates have been identified (Burgasser et al. 2006a; Warren et al. 2007; Delorme et al. 2008; Burningham et al. 2008) with estimated $T_{\text {eff }}$ extending below $600 \mathrm{~K}$ (e.g., ULAS J133553.45+113005.2). However, none of these sources have trigonometric parallax and broadband spectral energy distribution (SED) measurements, so such estimates must be treated with caution.

In this Letter, we present new mid-infrared (MIR) and astrometric observations of the T8 brown dwarf 2MASS J09393548-2448279 (hereafter 2M0939; Tinney et al. 2005) that allow us to determine its luminosity and constrain its atmospheric parameters. We find this source to have a luminosity comparable to $2 \mathrm{M} 0415$ but a $T_{\text {eff }}$ that is roughly $100 \mathrm{~K}$ colder,

\footnotetext{
${ }^{1}$ Massachusetts Institute of Technology, Kavli Institute for Astrophysics and Space Research, Building 37, Room 664B, 77 Massachusetts Avenue, Cambridge, MA 02139; ajb@mit.edu.

${ }^{2}$ Department of Astrophysics, School of Physics, University of New South Wales, NSW 2052, Australia.

${ }^{3}$ Institute for Astronomy, University of Hawaii, 2680 Woodlawn Drive, Honolulu, HI 96822.

${ }^{4}$ Los Alamos National Laboratory, Applied Physics Division, MS F663, Los Alamos, NM 87545.

${ }^{5}$ NASA Ames Research Center, MS 254-3, Moffett Field, CA 94035.

${ }^{6}$ Infrared Processing Analysis Center, MS 100-22, California Institute of Technology, Pasadena, CA 91125.
}

suggesting that 2M0939 may actually be the coldest and lowest luminosity unresolved pair of brown dwarfs identified to date.

\section{OBSERVATIONS}

The brown dwarf 2M0939 was observed with the Spitzer Space Telescope Infrared Array Camera (IRAC; Fazio et al. 2004) and Infrared Spectrograph (IRS; Houck et al. 2004) as part of program GO-20544 on 2005 December 25 and 2006 May 24 (UT), respectively. Five dithered IRAC exposures of $30 \mathrm{~s}$ each were obtained in all four photometric bands. Aperture photometry was measured on individual basic calibrated data (BCD) IRAC images produced by the Spitzer IRAC pipeline (ver. S14.0.0) using IRAF ${ }^{7}$ PHOT. Apertures of 5 pixels were used, with aperture corrections and Vega zero points adopted from the IRAC Data Handbook ${ }^{8}$ (see also Reach et al. 2005). From these data we determined [3.6] $=13.76 \pm 0.02,[4.5]=11.66 \pm$ $0.02,[5.8]=12.96 \pm 0.03$, and $[8.0]=11.89 \pm 0.03$, where uncertainties reflect the standard deviation of individual BCD measurements.

IRS spectra were obtained in the short-low (SL) mode, covering 5.3-15.3 $\mu \mathrm{m}$ at $\lambda / \Delta \lambda \approx 90$ in two orders. A total of 15 dithered pairs of $240 \mathrm{~s}$ exposures each (7200 s net integration) were obtained in both orders. Data were reduced as described in Cushing et al. (2006). The resulting spectrum was flux calibrated to IRAC [8.0]-band photometry, including a correction factor as required by the definition of the IRAC photometric system (Cushing et al. 2006). The median signal-to-noise ratio of the IRS spectrum was $\sim 40$, increasing to $\sim 160$ at the $10 \mu \mathrm{m}$ peak. These data are shown in Figure 1.

Astrometric observations of 2M0939 were obtained with the IRIS2 NIR camera and spectrograph (Tinney et al. 2004) on the $3.9 \mathrm{~m}$ Anglo-Australian Telescope. Ten observations were

\footnotetext{
${ }^{7}$ The Image Reduction and Analysis Facility (IRAF) is distributed by the National Optical Astronomy Observatory, which is operated by the Association of Universities for Research in Astronomy, Inc., under cooperative agreement with the National Science Foundation.

${ }^{8}$ See http://ssc.spitzer.caltech.edu/irac/dh/iracdatahandbook3.0.pdf.
} 

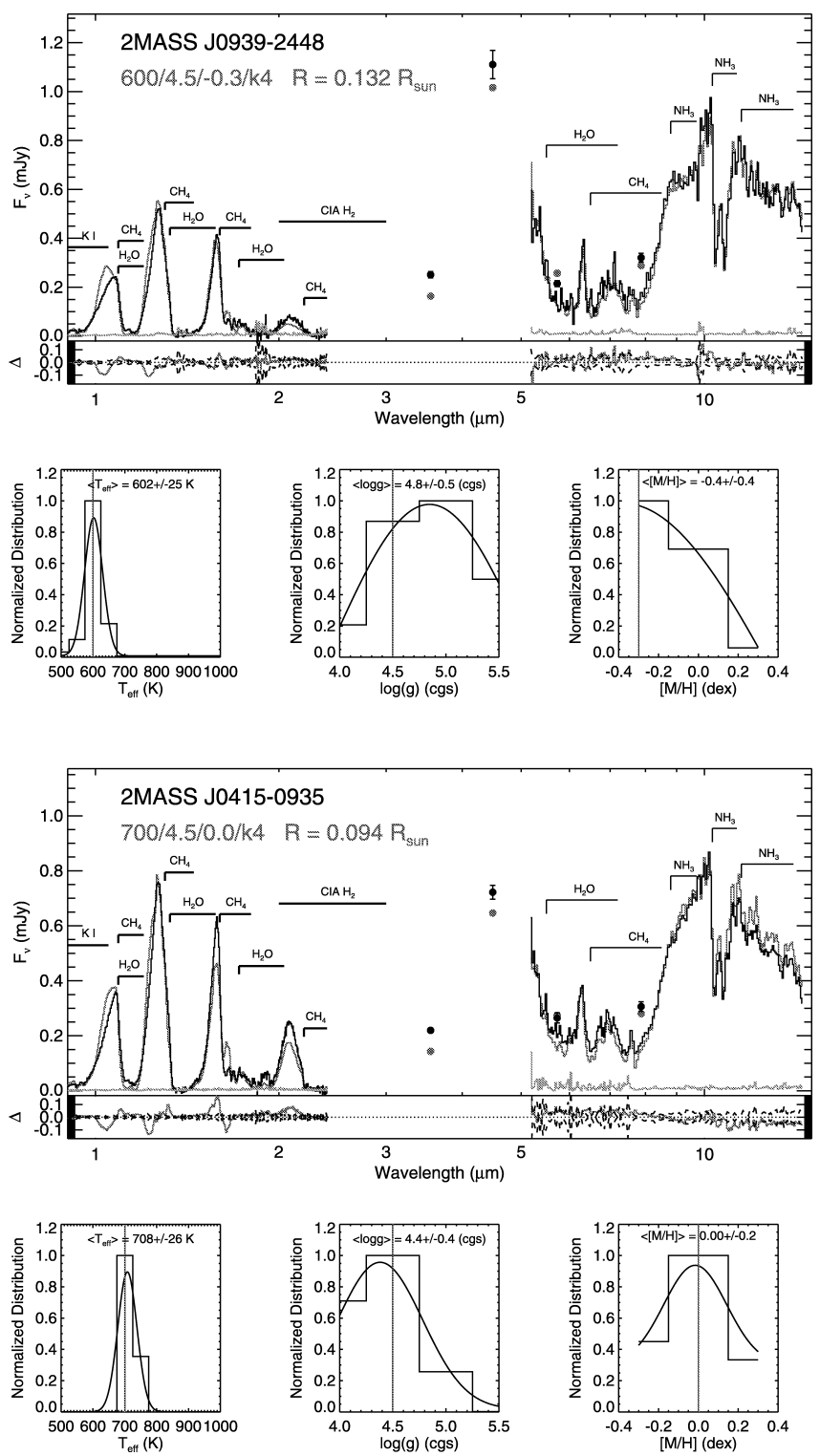

FIG. 1.-Best-fit spectral models (red lines) to absolutely flux-calibrated NIR and MIR data (black lines; uncertainties in gray) for 2M0939 (top) and 2M0415 (bottom). Major absorption features are noted. Also shown are observed (black points with error bars) and model (red points) IRAC fluxes. Fit residuals (red lines; data minus model) are shown at the bottom of each panel and are compared to \pm 3 times the observational uncertainty (dashed lines). Below these panels are the weighted distributions of $T_{\text {eff }}, \log g$, and $[\mathrm{M} / \mathrm{H}]$ parameters from the spectral model fits. Gaussian fits to the temperature distributions indicate $T_{\text {eff }}=602 \pm 25$ and $708 \pm 26 \mathrm{~K}$ for $2 \mathrm{M} 0939$ and $2 \mathrm{M} 0415$, respectively. [See the electronic edition of the Journal for a color version of this figure.]

conducted at seven epochs from 2005 January 8 to 2008 May 24. Each observation consisted of multiple, dithered $60 \mathrm{~s}$ exposures obtained through IRIS2's $\mathrm{MKO}^{9} J$-band filter, with the number of exposures depending on seeing conditions. Imaging data were reduced using a modified version of the ORAC-DR data reduction pipeline ${ }^{10}$ as described in Tinney et al. (2005). Astrometry of 2M0939 was tied to 11 reference stars in the $7.7^{\prime} \times 7.7^{\prime}$ field of view. A small correction from relative to absolute parallax $(0.7 \pm 0.4$ mas $)$ was determined by estimat-

\footnotetext{
${ }^{9}$ Mauna Kea Observatory filter system (Simons \& Tokunaga 2002; Tokunaga et al. 2002).

${ }^{10}$ See http://www.oracdr.org.
}

ing the mean parallax of the reference stars using a linear color/ magnitude relation $\left(M_{J}=1.74+3.92[I-J], \sigma=0.4 \mathrm{mag}\right)$ derived from the Catalogue of Nearby Stars ${ }^{11}$ and $I$-band photometry from the DENIS DR3 catalog (Epchtein et al. 1997). We also computed $J_{\text {MKO }}=15.566 \pm 0.011$ for $2 \mathrm{M} 0939$ by transforming 2MASS photometry (Skrutskie et al. 2006) of $J-K_{s}<1.0$ sources in the field of view onto the MKO system (Carpenter 2001).

At a distance $d=5.34 \pm 0.13 \mathrm{pc}(\pi=187.3 \pm 4.6 \mathrm{mas})$, 2M0939 is the fifth closest brown dwarf to the Sun currently known. Its relatively high proper motion $\left(\mu_{\alpha} \cos \delta=\right.$ $573.4 \pm 2.3$ mas $\left.\mathrm{yr}^{-1}, \mu_{\delta}=-1044.7 \pm 2.5 \mathrm{mas} \mathrm{yr}^{-1}\right)$ indicates a tangential velocity $\left(V_{\tan }=30.2 \pm 0.7 \mathrm{~km} \mathrm{~s}^{-1}\right)$ that is comparable to that of the majority of nearby late-type dwarfs (Faherty et al. 2008) and consistent with membership in the Galactic disk population. Its inferred absolute $J_{\text {мко }}$ magnitude, $M_{J_{\mathrm{MКо}}}=16.92 \pm 0.05$, is $0.4 \mathrm{mag}$ fainter than that of $2 \mathrm{M} 0415$ (Knapp et al. 2004; Vrba et al. 2004) and the lowest measured to date. However, 2M0939 is brighter than 2M0415 in the IRAC [3.6] and [4.5] bands, by over $0.4 \mathrm{mag}$ in the latter $\left(M_{[4.5]}=\right.$ $13.03 \pm 0.06$ versus $13.49 \pm 0.04$; Patten et al. 2006). Reddening, perhaps indicative of a circumsubstellar disk, does not appear to be responsible for this flux reversal, as 2M0939 is fainter in the [5.8] band and comparably bright at $8 \mu \mathrm{m}$.

\section{THE PHYSICAL PROPERTIES OF 2MASS J09393548-2448279}

\subsection{Luminosity}

The bolometric luminosity of 2M0939 was determined by integrating absolute flux-calibrated NIR (Burgasser et al. 2006a) and MIR spectral data, and interpolating unobserved spectral regions with photometrically calibrated spectral models. For this we employed three condensate-free spectral models from Allard et al. (2001), Burrows et al. (2006), and M. S. Marley et al. (in preparation) in an effort to gauge systematic effects. Our baseline calculations used models with parameters $T_{\text {eff }}=700 \mathrm{~K}$, surface gravity $\log g=5.0$ (cgs), and metallicity $[\mathrm{M} / \mathrm{H}]=0$, with the parameter $K_{z z}=10^{4} \mathrm{~cm}^{2} \mathrm{~s}^{-1}$ adopted for the Marley et al. model (see below). At short and long wavelengths, model spectra were scaled to match the ends of the NIR and MIR observational data, while the $2.4-5.2 \mu \mathrm{m}$ model spectra were split at $3.9 \mu \mathrm{m}$ and scaled separately to IRAC [3.6]- and [4.5]-band photometry. The calibration of the 3.9$5.2 \mu \mathrm{m}$ region was particularly important, as it constitutes roughly $45 \%$ of the total flux from this source. Fortunately, differences in the integrated flux between the models were less than $3 \%$ in this region. We also found $<1 \%$ differences in bolometric fluxes when model parameters were varied by $\Delta T_{\text {eff }}= \pm 100 \mathrm{~K}, \Delta \log g= \pm 0.5$, and $\Delta[\mathrm{M} / \mathrm{H}]= \pm 0.3$. Integrating over the combined SED, we find $\log _{10}\left(L_{\text {bol }} / L_{\odot}\right)=$ $-5.69 \pm 0.03$ for $2 \mathrm{M} 0939$, where the uncertainty includes distance and photometric uncertainties and differences between the three models. This luminosity is comparable to that of $2 \mathrm{M} 0415$, and for a nominal estimate of its radius $\left(R \sim 0.1 R_{\odot}\right)$ indicates $T_{\text {eff }} \approx 700 \mathrm{~K}$, roughly consistent with prior estimates (Burgasser et al. 2006a; Leggett et al. 2007).

\subsection{Spectral Model Fits}

The atmospheric properties of 2M0939 were determined by comparing the absolute flux-calibrated NIR and MIR spectral data to a set of theoretical spectral models from M. S. Marley

\footnotetext{
${ }^{11}$ Compiled by I. N. Reid; see http://www.stsci.edu/inr/cmd.html.
} 


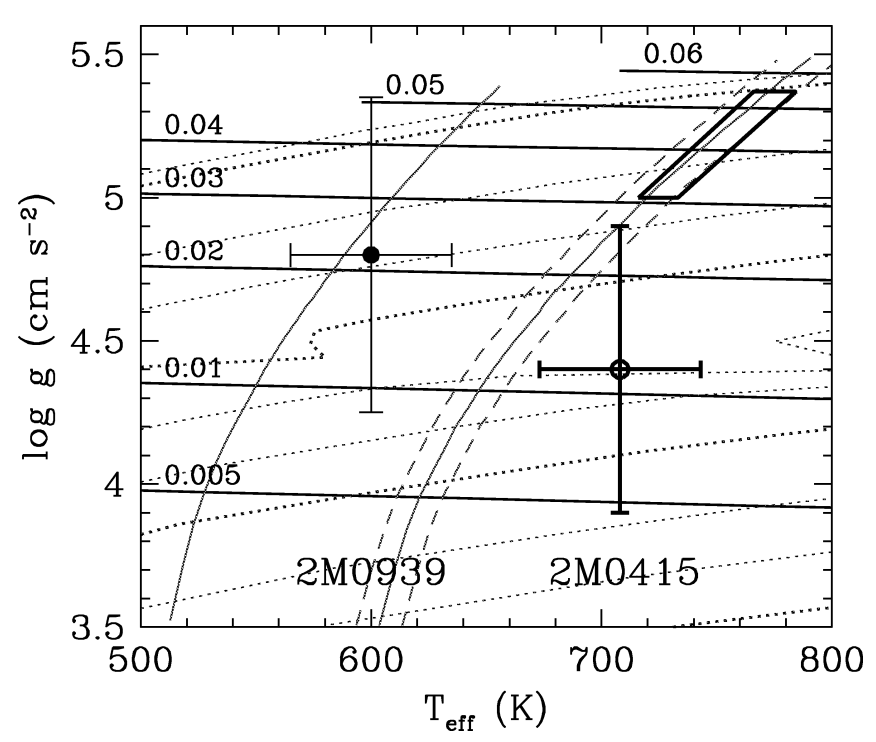

FIG. 2.-The $T_{\text {eff }}$ vs. $\log g$ evolutionary tracks for cloudless brown dwarfs with $[\mathrm{M} / \mathrm{H}]=-0.3$, from Saumon \& Marley (2008). Lines of constant mass are indicated by thick black lines. Isochrones (for values $0.01,0.02,0.04,0.1$, $0.2,0.4,1,2,4,10$, and $12 \mathrm{Gyr}$ from bottom to top) are indicated by dotted lines. The inferred $T_{\text {eff }}$ and $\log g$ of $2 \mathrm{M} 0939$ are indicated by the filled circle with error bars (which include systematic uncertainties). Its measured luminosity, $\log _{10}\left(L_{\mathrm{bol}} / L_{\odot}\right)=-5.69 \pm 0.03$, traces the locus indicated by the three red lines at right (dashed lines delineate the $1 \sigma$ uncertainty range). If 2M0939 is an equal-mass binary, the corresponding luminosity of each component traces the locus at left. Also shown are inferred $T_{\text {eff }}$ and $\log g$ values for $2 \mathrm{M} 0415$ from this Letter (open circle with error bars) and Saumon et al. (2007; parallelogram). [See the electronic edition of the Journal for a color version of this figure.]

et al. (in preparation; see also Cushing et al. 2008). We focused on models without condensate cloud opacity, as late-type $\mathrm{T}$ dwarf photospheres appear to be relatively cloud-free (e.g., Allard et al. 2001), although elemental depletion due to condensation is included in the chemistry (Freedman et al. 2008). We explicitly considered nonequilibrium mixing via vertical transport as parameterized by a vertical diffusion coefficient $K_{z z}$ (Saumon et al. 2006). In all, we examined 262 models spanning parameters $T_{\text {eff }}=500-1000 \mathrm{~K} \quad(50 \mathrm{~K}$ steps $)$; $\log g=4.0-5.5(0.5$ dex steps $) ;[\mathrm{M} / \mathrm{H}]=-0.3,0,+0.3$; and $K_{z z}=0,10^{4} \mathrm{~cm}^{2} \mathrm{~s}^{-1}$. The quality of fit between the spectral data and models was quantified using a goodness-of-fit statistic, $G_{k}$, as defined in Cushing et al. (2008). We chose a weighting scheme that forced better fitting of regions most sensitive to $T_{\text {eff }}, \log g$, and [M/H] (Cushing et al. 2008). We also heavily weighted (by a factor of 10) the MIR spectral data due to persistent incompleteness in $\mathrm{CH}_{4}$ and $\mathrm{NH}_{3}$ opacities shortward of $1.7 \mu \mathrm{m}$ (Saumon et al. 2007), and excluded the 1.6-1.8 $\mu \mathrm{m}$ $\mathrm{CH}_{4}$ band from the fits. The best-fit normalization of the model, derived by minimizing $G_{k}$, is equivalent to $(R / d)^{2}$ and hence provides an independent radius determination.

Figure 1 displays the best-fitting model (smallest $G_{k}$ ) for 2M0939, with parameters $T_{\text {eff }}=600 \mathrm{~K}, \quad \log g=4.5$, $[\mathrm{M} / \mathrm{H}]=-0.3$, and $K_{z z}=10^{4} \mathrm{~cm}^{2} \mathrm{~s}^{-1}$. This model provides a good match to the broadband SED, with detailed fits of the deep $\mathrm{H}_{2} \mathrm{O}, \mathrm{CH}_{4}$, and $\mathrm{NH}_{3}$ bands particularly in the MIR, where residuals differ by less than 3 times the observational uncertainty. The strong $\mathrm{NH}_{3}$ band at $10.5 \mu \mathrm{m}$, as well as distinct features at 9 and $12.5 \mu \mathrm{m}$, are all reproduced in detail. $\mathrm{NH}_{3}$ absorption is highly sensitive to nonequilibrium mixing in these models, so our nonzero best-fit value for $K_{z z}$ supports the ubiquity of such mixing in late-type T dwarf photospheres (Saumon et al. 2006, 2007). The NIR fit is not as good, diverging considerably at the $1.05 \mu \mathrm{m}$ flux peak. This peak is shaped by the pressure-broadened red wing of the $0.77 \mu \mathrm{m} \mathrm{K}$ I doublet, for which more recent calculations are not incorporated in the models (e.g., Burrows \& Volobuyev 2003). Note also that model [3.6]-band fluxes (red circles) are somewhat fainter than observed.

The best-fit $T_{\text {eff }}$ value of $600 \mathrm{~K}$ is surprising given the luminosity measurement above, requiring a radius $\left(R=0.13 R_{\odot}\right)$ that is considerably larger than expected from brown dwarf structure models (e.g., Saumon \& Marley 2008). Yet warmer models with higher surface fluxes fail to reproduce the SED of 2M0939, with relatively brighter NIR fluxes and weaker $\mathrm{NH}_{3}$ absorption. Variations in the other model parameters to allow warmer $T_{\text {eff }}$ did not change this conclusion. To quantify our uncertainties, we constructed weighted distributions of $T_{\text {eff }}, \log g$, and $[\mathrm{M} / \mathrm{H}]$ in which each model's parameters are weighted by the factor ${ }^{12} e^{-0.1 G_{k}}$. Figure 1 shows the weighted distribution of $T_{\text {eff }}$ values; a Gaussian fit yields $\left\langle T_{\text {eff }}\right\rangle=$ $602 \pm 25 \mathrm{~K}$, consistent with the single best-fit model. Similarly, we find $\langle\log g\rangle=4.8 \pm 0.5$ and $\langle[\mathrm{M} / \mathrm{H}]\rangle-0.4 \pm 0.4$ (i.e., subsolar metallicities are highly favored). To these uncertainties we include systematic uncertainties of $\pm 25 \mathrm{~K}$, $\pm 0.25 \mathrm{dex}$, and $\pm 0.15 \mathrm{dex}$, respectively, to account for parameter sampling.

To place these results in context, we also fit equivalent NIR and MIR data for 2M0415 (Burgasser et al. 2004; Saumon et al. 2007) following the same procedures. The best-fit model for this source (Fig. 1) has parameters $T_{\text {eff }}=700 \mathrm{~K}$, $\log g=4.5,[\mathrm{M} / \mathrm{H}]=0$, and $K_{z z}=10^{4} \mathrm{~cm}^{2} \mathrm{~s}^{-1}$, with a more reasonable radius determination $\left(R=0.094 R_{\odot}\right)$. The fit in the MIR is not as good as that for 2M0939, deviating from $\pm 3 \sigma$ for $\lambda>10 \mu \mathrm{m}$. Saumon et al. (2007) have shown that a slightly warmer $T_{\text {eff }}=730 \mathrm{~K}$ model is better in this region. Our weighted parameter values are nevertheless roughly consistent with those of Saumon et al. (2007) with $\left\langle T_{\text {eff }}\right\rangle=708 \pm 26 \mathrm{~K}$, $\langle\log g\rangle=4.4 \pm 0.4$ (less than that inferred by Saumon et al.), and $\langle[\mathrm{M} / \mathrm{H}]\rangle=0.0 \pm 0.2$.

We conclude that $2 \mathrm{M} 0939$ is roughly $100 \mathrm{~K}$ cooler than 2M0415, despite having a similar luminosity. This is consistent with the overall redder SED and deeper $\mathrm{NH}_{3}$ bands of 2M0939, differences that cannot be attributed to gravity or metallicity effects (see also Saumon et al. 2007). The low $T_{\text {eff }}$ of $2 \mathrm{M} 0939$ is also consistent with its red $H-$ [4.5] color, based on the linear correlation found by Warren et al. (2007) for latetype $\mathrm{T}$ dwarfs. We find that 2M0939 $(H-[4.5]=4.27 \pm$ $0.05)$ is redder than both $2 \mathrm{M} 0415(H-[4.5]=3.42 \pm 0.03)$ and ULAS J003402.77-005206.7 $(H-[4.5]=4.00 \pm 0.05$, $T_{\text {eff }}=660 \pm 15 \mathrm{~K}$; Warren et al. 2007), and comparable in color to ULAS J133553.45+113005.2 $(H-[4.5]=4.34 \pm$ $0.04, T_{\text {eff }}=575 \pm 25 \mathrm{~K}$; Burningham et al. 2008).

\section{DISCUSSION}

Figure 2 illustrates how the low $T_{\text {eff }}$ of $2 \mathrm{M} 0939$ is difficult to reconcile with its measured luminosity based on evolutionary models from Saumon \& Marley (2008). The best-fit value of $T_{\text {eff }}=600 \mathrm{~K}$ can only be reproduced for the lowest mass $\left(M<0.001 M_{\odot}\right)$, youngest $(\tau<20 \mathrm{Myr})$, and lowest surface gravity $(\log g<3.5)$ tracks, in clear disagreement with the

\footnotetext{
${ }^{12} G_{k}$ is similar in form to $\chi^{2}$, so we adopt an exponential form for the probability distribution. The 0.1 factor in the exponent was a conservative value chosen to reflect clear degradation in the fits when examined by eye.
} 
$\log g$ derived from spectral modeling and in seeming disagreement with the kinematics of this source. We do not believe that this disagreement indicates erroneous parameters from the spectral model fits given the excellent quality of the fits and the fact that neither warmer nor lower surface gravity models can reproduce the data. Structure models are also unlikely to substantially underestimate the radii of evolved brown dwarfs given the excellent agreement for other late-type T dwarfs such as 2M0415 (Saumon et al. 2006, 2007).

We consider instead the possibility that $2 \mathrm{M} 0939$ is overluminous for its photospheric $T_{\text {eff }}$ because it is an unresolved binary system. Roughly $20 \%$ of all field brown dwarfs are binaries, the vast majority being near equal-mass/equal-brightness systems (Allen 2007). In such a case, the inferred $T_{\text {eff }}$ of each component of the binary would be equal to that derived from the spectral model fits above (as determined by the shape of the spectrum), but luminosities would be scaled down by half to $\log _{10}\left(L_{\mathrm{bol}} / L_{\odot}\right) \approx-6$. Figure 2 displays the loci of allowed $T_{\text {eff }}$ and $\log g$ values for this lower luminosity, for which fit parameters are consistent with $M \approx 0.01-0.05 M_{\odot}$ and an age in the range 0.4-12 Gyr. These are in better agreement with the expected properties of 2M0939, particularly its kinematics. Note also that the inferred radii for binary components would scale by a factor of $1 / \sqrt{2}$, matching the radius of 2M0415 and current predictions by brown dwarf structure models. Finally, unresolved multiplicity would explain why 2M0939 appears fainter than 2M0415 in the NIR but brighter in the MIR; each component of a binary 2M0939 would in fact be fainter than 2M0415 at all wavelengths.

The binary hypothesis thus provides a reasonable explana- tion for the disagreement between the derived $T_{\text {eff }}$ and luminosity of 2M0939 and is testable via high-resolution imaging and/or radial velocity monitoring observations.

We note that Goldman et al. (2008) did not resolve this source in recent ground-based adaptive optics imaging. This indicates that a binary 2M0939 would have to have a separation $\lesssim \$ 0.5$ AU (making it a prime target for radial velocity monitoring), or it was observed close to alignment (cf. Kelu-1; Liu \& Leggett 2005; Gelino et al. 2006). Additional high-resolution imaging epochs are needed to rule out 2M0939 as a resolved pair. The very low luminosity and $T_{\text {eff }}$ of this source, in addition to its possible multiplicity, warrants further investigation.

This publication makes use of data from the Two Micron All Sky Survey (2MASS), which is a joint project of the University of Massachusetts and the Infrared Processing and Analysis Center (IPAC), and funded by NASA and the NSF. The 2MASS data were obtained from the NASA/IPAC Infrared Science Archive, which is operated by the Jet Propulsion Laboratory, California Institute of Technology (JPL/Caltech), under contract with NASA. This work is also based in part on observations made with the Spitzer Space Telescope, which is operated by JPL/Caltech under a contract with NASA. Support for this work was provided by NASA through an award issued by JPL/Caltech. C. G. T. acknowledges support from ARC grant DP0774000. Support for D. S.'s work, part of the Spitzer Space Telescope Theoretical Research Program, was provided by NASA. We thank our anonymous referee for her/his prompt review of our original manuscript.

\section{REFERENCES}

Allard, F., Hauschildt, P. H., Alexander, D. R., Tamanai, A., \& Schweitzer, A. 2001, ApJ, 556, 357

Allen, P. R. 2007, ApJ, 668, 492

Burgasser, A. J., Burrows, A., \& Kirkpatrick, J. D. 2006a, ApJ, 639, 1095

Burgasser, A. J., Geballe, T. R., Leggett, S. K., Kirkpatrick, J. D., \& Golimowski, D. A. 2006b, ApJ, 637, 1067

Burgasser, A. J., McElwain, M. W., Kirkpatrick, J. D., Cruz, K. L., Tinney, C. G., \& Reid, I. N. 2004, AJ, 127, 2856

Burgasser, A. J., et al. 2002, ApJ, 564, 421

Burningham, B., et al. 2008, MNRAS, in press

Burrows, A., Sudarsky, D., \& Hubeny, I. 2006, ApJ, 640, 1063

Burrows, A., \& Volobuyev, M. 2003, ApJ, 583, 985

Carpenter, J. M. 2001, AJ, 121, 2851

Cushing, M. C., et al. 2006, ApJ, 648, 614

- 2008, ApJ, 678, 1372

Delorme, P., et al. 2008, A\&A, 482, 961

Epchtein, N., et al. 1997, Messenger, 87, 27

Faherty, J. K., Burgasser, A. J., Cruz, K. L., Shara, M. M., Walter, F. M., \& Gelino, C. R. 2008, AJ, in press

Fazio, G., et al. 2004, ApJS, 154, 10

Freedman, R. S., Marley, M. S., \& Lodders, K. 2008, ApJS, 174, 504
Gelino, C. R., Kulkarni, S. R., \& Stephens, D. C. 2006, PASP, 118, 611 Goldman, B., Bouy, H., Zapatero Osorio, M. R., Stumpf, M. B., Brandner, W., \& Henning, T. 2008, A\&A, 490, 763

Houck, J. R., et al. 2004, ApJS, 154, 18

Knapp, G., et al. 2004, AJ, 127, 3553

Leggett, S. K., et al. 2007, ApJ, 667, 537

Liu, M. C., \& Leggett, S. K. 2005, ApJ, 634, 616

Patten, B. M., et al. 2006, ApJ, 651, 502

Reach, W. T., et al. 2005, PASP, 117, 978

Saumon, D., \& Marley, M. 2008, ApJ, in press

Saumon, D., Marley, M. S., Cushing, M. C., Leggett, S. K., Roellig, T. L., Lodders, K., \& Freedman, R. S. 2006, ApJ, 647, 552

Saumon, D., et al. 2007, ApJ, 656, 1136

Simons, D. A., \& Tokunaga, A. T. 2002, PASP, 114, 169

Skrutskie, M. F., et al. 2006, AJ, 131, 1163

Tinney, C. G., Burgasser, A. J., Kirkpatrick, J. D., \& McElwain, M. W. 2005, AJ, 130, 2326

Tinney, C. G., et al. 2004, Proc. SPIE, 5492, 998

Tokunaga, A. T., Simons, D. A., \& Vacca, W. D. 2002, PASP, 114, 180

Vrba, F. J., et al. 2004, AJ, 127, 2948

Warren, S. J., et al. 2007, MNRAS, 381, 1400 